\title{
Eorrosión de armaduras en los hormigones armados y pretensados
}

\section{sinopsis}

\author{
J. C A L LE J A*
}

Dentro del capitulo general de la "durabilidad" o resistencia química en el tiempo de los conglomerados hidráulicos (morteros y hormigones armados y pretensados), el apartado relativo a la "corrosión" de las armaduras ocupa un lugar preponderante por su importancia técnica y económica.

Esta importancia va siendo reconocida cada día más, a pesar de ser (o de haber sido) el de la corrosión uno de los temas menos familiares en el ámbito de la construcción. La razón estriba en la fuerza de los hechos, y se manifiesta con claridad en las tareas encomendadas y en los problemas presentados a los laboratorios de investigación y ensayos de materiales.

La corrosión es un fenómeno fisicoquímico que cae dentro del dominio de la ELECTROQUIMICA y que debe estudiarse y enjuiciarse con arreglo a las leyes fundamentales de esta disciplina. Lo mismo cabe decir por lo que respecta a los métodos de "protección" contra la corrosión.

La corrosión es un "riesgo" aleatorio que se corre y contra el que hay que prevenirse mediante un "seguro", representado por dichas medidas de protección.

El remedio "más fácil" desde un punto de vista técnico, y "más barato" desde un punto de vista "económico", es siempre de carácter "preventivo" antes que "curativo", es decir, es el tomado ya en la etapa de proyecto de la obra o estructura de que se trate.

En el caso del hormigón, la mejor protección de las armaduras la da, en primer lugar, la aplicación de un "código de buena práctica" a la elaboración del material y a la ejecución de la obra, y, en segundo lugar, la observancia de los más elementales principios de la Electroquímica en el aspecto de la corrosión.

La utilización de aditivos acelerantes de fraguado y endurecimiento con naturaleza "clorurada" y el estado de "tensión" de las armaduras son aspectos que merecen especial consideración, así como las posibles acciones electroquímicas debidas a "corrientes vagabundas" a través de las armaduras de la estructura.

\section{0. antecedentes}

El problema de la corrosión metálica bien puede calificarse como de plaga, por cuanto que sus desfavorables repercusiones técnicas y económicas son extraordinarias. Para darse idea de ello bastaría consultar las estadísticas de las destrucciones anuales producidas en el mundo, en obras y estructuras metálicas de todo tipo, a causa de la corrosión. Estas destrucciones ascienden a millones de toneladas y, paralelamente, las pérdidas se elevan a miles de millones de pesetas.

De aqui que se haya desarrollado tanto en los últimos tiempos el estudio de los métodos de protección contra la corrosión, basados en el conocimiento del fenómeno, de sus causas y del modo de producirse en cada caso.

- Doctor en Ciencias Quimicas. Investigador Jefe del Departamento de Ouímica del Instituto Eduar. do Torroja de la Construcción y del Cemento, Patronato "Juan de la Cierva" de Investigación Científica y Técnica, Consejo Superior de Investigaciones Científicas. 
En la construcción intervienen elementos y estructuras metálicas puras, con las que se siguen estos métodos de protección. Pero intervienen también estructuras y elementos mixtos, en que los metales (y concretamente el hierro y el acero) se alían con otros materiales pétreos $\mathrm{y}$, en particular, con conglomerantes hidráulicos. Por ello, en muchas ocasiones, el acero se encuentra sometido a acciones y fuerzas de tipo mixto, físico y químico, que implican formas especiales o específicas de corrosión.

Si bien los casos de corrosión de esta clase "registrados" no son muchos, son en cambio muchísimos los que se dan real y prácticamente. Cada día es mayor la justa preocupación, por ser mayores el conocimiento del tema y la difusión del mismo. Así lo pueden atestiguar los laboratorios de ensayos de materiales, donde van siendo frecuentes las consultas sobre esta materia. Así lo confirman los ensayos realizados y los informes emitidos por el Departamento de Química del Instituto Eduardo Torroja de la Construcción y del Cemento.

La corrosión es una enfermedad casi siempre fácil de prevenir y a veces muy difícil de curar. Y donde se dice fácil y difícil, puede leerse también barato y caro. Sería aconsejable para los técnicos de la construcción el que, con la misma asiduidad y rutina con que en el proyecto de las obras consideran capítulos tales como la resistencia de la estructura, la impermeabilidad, la durabilidad, etc., considerasen igualmente el de la corrosión metálica de todo tipo (incluible en el último de los capítulos citados). Y esto por razones técnicas y económicas.

El problema de la corrosión va estrechamente ligado a una parte muy concreta de la Química Física: la ELECTROQUIMICA, en la cual se asientan las bases teóricas y prácticas de su estudio. También en ella se apoyan los principios de la protección contra la corrosión, por lo que, ante casos de vcrdadera importancia por su volumen o responsabilidad, no debe dudarse en recurrir al dictamen o consejo del especialista en dicha disciplina. Y, si es posible, preferentemente a la hora del proyecto, mejor que a la hora de la realización o a la del primer fallo debido a la corrosión.

\section{1. introducción}

El hormigón es un conglomerante pétreo artificial formado por unos áridos pétreos naturales, generalmente considerados como inertes, aunque no siempre con razón, un conglomerante activo (cemento), agua y, eventualmente, alguna adición en pequeña dosis para conseguir una cierta propiedad o comportamiento deseados.

El hormigón constituye un material estructural cuya misión es resistir y durar. Resistir mecánicamente los esfuerzos para los que está calculado, y durar lo más posible en el tiempo y en las condiciones de servicio, sin perder ninguna de sus propiedades y, en particular, las mecánico-resistentes.

Así, pues, resistencia mecánica y durabilidad, durabilidad y resistencia mecánica, son las dos características más destacadas del hormigón.

La resistencia mecánica depende, en forma compleja, de una serie de factores que no es del caso analizar.

La durabilidad depende, a su vez, de las interacciones entre el hormigón y el ambiente particular en que éste se encuentra. Estas interacciones son, en general, de naturaleza quí- 
mica, por lo cual la durabilidad del hormigón puede considerarse como su resistencia o su capacidad de resistencia quimica.

Las interacciones entre el hormigón y su medio ambiente guardan relación muy compleja con las características de uno y otro y, en particular, incluso con la resistencia mecánica del primero.

El hormigón, tal y como se ha definido, puede considerarse, en cierto modo, como un material homogéneo. Su evolución, tendente a conseguir cada vez mayores resistencias mecánicas con menores secciones, por motivos estéticos o económicos, ha dado lugar a la alianza del material pétreo con un material metálico, el acero, resultando de ella unos conglomerados heterogéneos, como son el hormigón armado y el hormigón pretensado.

La mejora en el aspecto de las resistencias mecánicas es evidente, y así lo prueba el amplio desarrollo experimentado por los citados materiales y las correspondientes técnicas.

Pero, en cuanto a la durabilidad de los mismos se refiere, entran en juego nuevos factores, ya que no se trata tan sólo de la relativa al material pétreo (hormigón), sino que hay que tener también en cuenta la del material metálico (armaduras de acero).

La durabilidad de las armaduras depende igualmente de las interacciones entre ellas y el ambiente particular en que se encuentren. Este ambiente puede ser directo o inmediato: el hormigón en cuya masa se hallan embebidas; e indirecto o exterior: el propio medio ambiente en que esté situado el hormigón.

Las interacciones en cuestión son, en general, de naturaleza electroquímica en el más amplio sentido, por lo cual la durabilidad de las armaduras en el hormigón puede considerarse como su resistencia o su capacidad de resistencia a la corrosión, ya que esta corrosión, como se verá, tiene generalmente carácter electroquímico. De aquí que el estudio de todo fenómeno de corrosión metálica caiga ineludiblemente dentro del terreno de la ELECTROQUIMICA. Es evidente que las citadas interacciones deben estar en relación íntima y muy compleja con las características del acero, con las del hormigón y con las del medio ambiente exterior.

La durabilidad del hormigón y la de los materiales metálicos constituyen de por sí, y por separado, amplios temas de sendas especialidades. Por ello, en esta publicación se intenta tratar, en exclusiva, de algunos aspectos, los más destacados, de la corrosión de las armaduras del hormigón y, en particular, de la influencia que en la misma ejerce la presencia de iones cloro.

\section{2. generalidades}

\section{1. mecanismo de la corrosión metálica.-clases de corrosión}

Intencionadamente se ha definido el hormigón armado como material o sistema heterogéneo, y es sabido que toda heterogeneidad es, cuando menos en potencia, asiento de fenómenos de corrosión, los cuales se dan en las superficies límite o de separación entre las fases homogéneas (interfases). 


\section{elementos primarios, secundarios y células electroliticas \\ (Nomenclatura)}

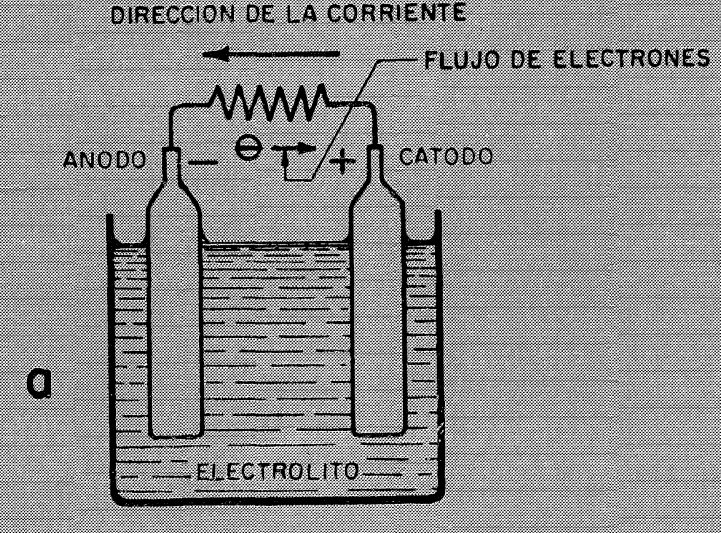

PILA GALVANICA

O ELEMENTO VOLTAICO

(PRIMARIO)

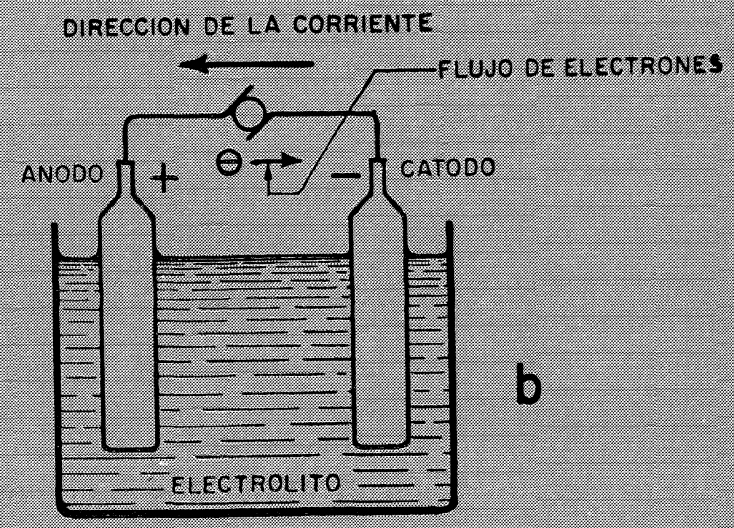

CELULA ELECTRONICA

DESCARGA

DIRECCION DE LA CORRIENTE

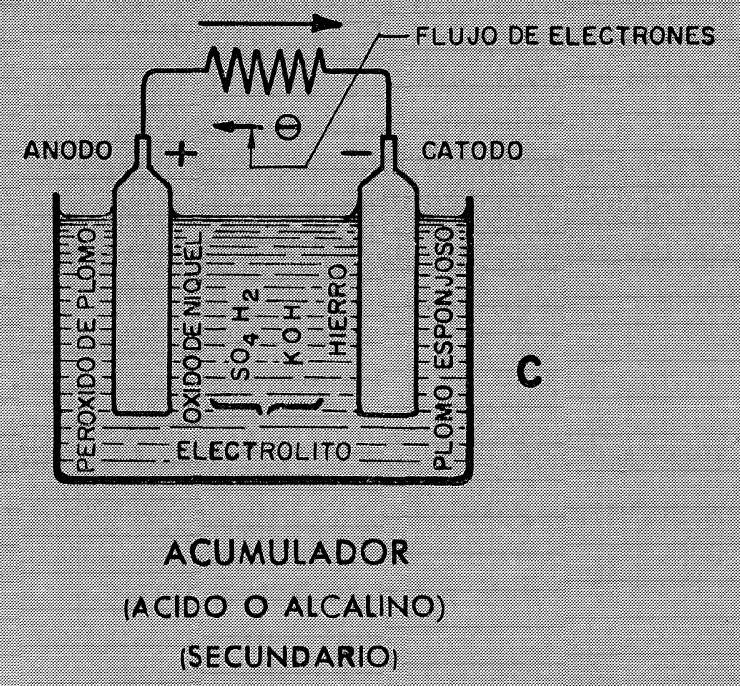

Fig. 1.

La corrosión metálica, desde el punto de vista que aquí interesa es, pues, una reacción heterogénea que tiene lugar en una interfase (la superficie límite o superficie de contacto entre las armaduras y la masa del hormigón), y que produce la destrucción del metal a partir de la superficie del mismo.

La corrosión metálica en general es debida a la tendencia de los metales a "volver a la 
Naturaleza", es decir, al estado en que se encuentran en las menas metálicas o minerales naturales de que proceden. Por supuesto que dicho estado es el más estable o de menor energía potencial, y que al sacar del mismo a los elementos metálicos por los procedimientos que brindan las distintas "metalurgias" para su obtención, se les confiere una energía potencial mayor y, por lo tanto, se les provoca una inestabilidad.

El mecanismo del proceso corrosivo responde a una oxidación en sentido generalizado, es decir, a una pérdida de electrones por parte del metal que se corroe. Por la forma en que este mecanismo de oxidación tiene lugar se ha querido distinguir entre oxidación o corrosión química y electroquímica, por una parte, y oxidación o corrosión general o uniforme, y localizada, por otra.

Corrosión química es la que se produce por interacción directa entre el metal y el medio ambiente, como, por ejemplo, la oxidación del hierro, en sentido restringido, por el oxígeno del aire y, en general, la reacción del hierro con gases secos que no sean electrólitos, es decir, que en disolución acuosa no den lugar por disociación a la formación de iones (átomos o agrupaciones atómicas con carga eléctrica negativa - aniones - o positiva - cationes--). La corrosión electroquímica implica una corriente eléctrica o desplazamiento de electrones de un lugar a otro del metal, lo cual puede suceder de manera espontánea y sin el concurso o aplicación de una fuerza electromotriz externa (creación de pilas o elementos galvánicos primarios), o con el concurso de una tal fuerza (creación de células electrolíticas). En todo caso, las reacciones primarias tienen lugar en los electrodos de dichos elementos o células, y la corrosión concretamente en el ánodo (convencionalmente el polo negativo en los elementos voltaicos o galvánicos, y el polo positivo en las células electroliticas) (fig. 1).

\section{2. causas de la corrosión metálica}

La citada tendencia de los metales a volver a su estado natural de combinaciones químicas tiene diversas manifestaciones, una de las cuales es la mayor o menor facilidad y rapidez de reacción de los metales con el agua y con los ácidos en condiciones distintas de temperatura, para dar lugar a diferentes tipos de procesos. Otra, la recíproca, es la mayor o menor facilidad de reducción de los respectivos óxidos metálicos con distintos agentes reductores, y la mayor o menor facilidad de descomposición de dichos óxidos por el calor.

Ordenados los metales más usuales de acuerdo con dicha facilidad de reacción o de reducción de sus óxidos, se obtiene la llamada serie electromotriz o serie electroquímica de los metales (cuadro 1), en la cual los elementos metálicos se sitúan por orden decreciente de actividad, es decir, de su tendencia a formar iones positivos (cationes) por pérdida de electrones (oxidación). Al orden decreciente de actividad corresponde un orden creciente de "nobleza".

Dicha ordenación no resulta solamente de una apreciación cualitativa de los hechos, sino que se apoya sobre una base cuantitativa: los valores de los potenciales electroquímicos normales de los elementos o, por mejor decir, de los electrodos o semielementos galvánicos constituidos por un metal, elemento químico en general, complejo iónico o molecular, y los iones simples o complejos a que en cada caso aquéllos den lugar. 
CUADro 1

SERIE ELECTROMOTRIZ DE LOS METALES

\begin{tabular}{|c|c|c|c|}
\hline Reaccionan con & Nombre & Símbolo & Los óxidos se reducen \\
\hline $\begin{array}{l}\text { Agua fría } \ldots \ldots \ldots \ldots \ldots \ldots \ldots \ldots \ldots \ldots \\
\text { Acidos (violentamente) } \ldots \ldots \ldots \ldots .\end{array}$ & $\left\{\begin{array}{l}\text { Litio } \\
\text { Potasio } \\
\text { Bario } \\
\text { Estroncio } \\
\text { Calcio } \\
\text { Sodio }\end{array}\right.$ & $\begin{array}{l}\mathrm{Li} \\
\mathrm{K} \\
\mathrm{Ba} \\
\mathrm{Sr} \\
\mathrm{Ca} \\
\mathrm{Na}\end{array}$ & $\begin{array}{l}\text { Ni por el hidrógeno, ni por el óxido } \\
\text { de carbono. }\end{array}$ \\
\hline & $\left(\begin{array}{l}\text { Magnesio } \\
\text { Aluminio } \\
\text { Manganeso } \\
\text { Zinc }\end{array}\right.$ & $\begin{array}{l}\mathrm{Mg} \\
\mathrm{Al} \\
\mathrm{Mn} \\
\mathrm{Zn}\end{array}$ & Por el carbón a alta temperatura. \\
\hline 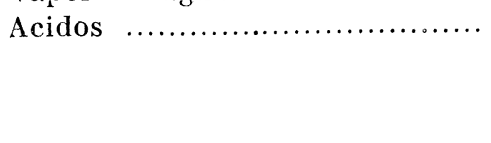 & $\begin{array}{l}\text { Hierro } \\
\text { Níquel } \\
\text { Estaño } \\
\text { Plomo }\end{array}$ & $\begin{array}{l}\mathrm{Fe} \\
\mathrm{Ni} \\
\mathrm{Sn} \\
\mathrm{Pb}\end{array}$ & \\
\hline & Hidrógeno & $\mathrm{H}$ & \\
\hline $\begin{array}{r}\text { Acidos oxidantes (sin despren- } \\
\text { der hidrógeno) } \ldots \ldots \ldots \ldots \ldots \ldots . . .\end{array}$ & $\left\{\begin{array}{l}\text { Cobre } \\
\text { Bismuto } \\
\text { Plata } \\
\text { Mercurio }\end{array}\right.$ & $\begin{array}{l}\mathrm{Cu} \\
\mathrm{Bi} \\
\mathrm{Ag} \\
\mathrm{Hg}\end{array}$ & $\begin{array}{l}\text { Por el hidrógeno y por el óxido de } \\
\text { carbono a alta temperatura. }\end{array}$ \\
\hline Agua regia........... & $\left\{\begin{array}{l}\text { Oro } \\
\text { Platino }\end{array}\right.$ & $\begin{array}{l}\mathrm{Au} \\
\mathrm{Pt}\end{array}$ & $\begin{array}{l}\text { Por simple calentamiento (descompo- } \\
\text { sición). }\end{array}$ \\
\hline
\end{tabular}

\section{3. origen del potencial eléctrico.-elementos y semielementos galvánicos}

Si un metal se pone en contacto con agua, la mencionada tendencia de sus átomos a convertirse en cationes y disolverse libera electrones sobre el metal. Por lo tanto, éste se carga negativamente. Por analogía con la tendencia de los líquidos a pasar al estado gaseoso, expresada y medida por la presión o tensión de vapor, la tendencia de los átomos metálicos a convertirse en cationes se ha llamado presión o tensión de disolución electrolitica.

Si un metal se pone en contacto con una disolución de sus propios cationes, además de la tendencia que da lugar a la presión de disolución electrolitica, actúa otra nueva tendencia: la que impele a los cationes a descargarse sobre el metal convirtiéndose en átomos neutros y cargando al metal positivamente. Esta nueva tendencia está expresada y medida por la presión osmótica de los cationes en la disolución.

Por lo tanto, en la inmersión de un metal en agua o en una disolución electrolítica de sus iones acaban por coexistir dos tendencias opuestas: la definida por la presión de disolución electrolitica que tiende a dar al metal una carga eléctrica negativa, y la definida por la presión osmótica que tiende a comunicarle una carga eléctrica positiva. Entre ambas tendencias se establece un equilibrio dinámico.

Estas cargas originan una doble capa eléctrica, pues los cationes del metal no pueden alejarse mucho dentro de la disolución y los electrones en exceso permanecen en la su- 
perficie del metal, dado que entre ambos portadores de carga se establece una mutua atracción electrostática.

Entre las dos partes de esta doble capa eléctrica (semejantes a las dos armaduras de un condensador eléctrico), y a través de ella, se establece una diferencia de potencial cuyo signo está determinado por la polaridad de la doble capa. Esta polaridad depende, a su vez, de los valores relativos de la presión de disolución del metal y de la presión osmótica de los cationes del mismo.

\section{dobles capas y cotenciales}
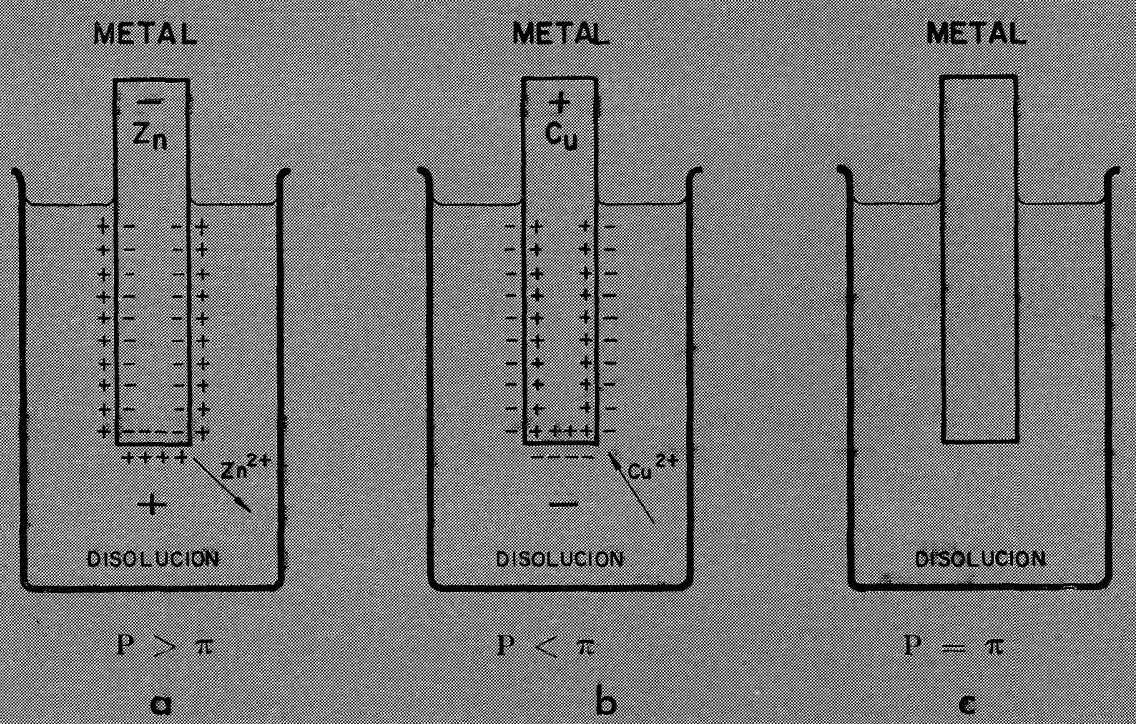

Iig. 2.

Si la presión de disolución electrolítica $P$ es mayor que la presión osmótica $\pi$, el metal se carga negativamente, y viceversa. Lo primero sucede con los metales situados en el extremo superior de la serie electromotriz (extremo negativo: $\mathrm{Zn}$, por ejemplo; fig. $2 a$ ); lo segundo ocurre con los situados en el extremo inferior (extremo positivo: Cu, por ejemplo; fig. $2 b$ ).

Si la presión de disolución electrolítica del metal $P$ es igual a la presión osmótica de sus iones $\pi$, no existe doble capa ni diferencia de cargas ni de potencial entre el metal y la disolución (fig. $2 c$ ).

Un metal en contacto con una disolución de sus iones constituye un semielemento galvánico o voltaico. La diferencia de potencial entre el metal y la disolución (diferencia de potencial a través de la doble capa) es el potencial aislado de dicho semielemento, y su signo es el correspondiente al metal. 
Un elemento voltaico, pila o célula galvánica primaria, es un sistema reaccionante formado por dos semielementos, en el que la energía química se transforma en energía eléctrica, con la correspondiente reducción de energía libre del sistema. La energía eléctrica depende de la fuerza electromotriz de la pila, esto es, de la diferencia de potencial entre los dos semielementos de que consta.

Esto quiere decir que el potencial aislado de un semielemento viene dado por la diferencia entre la fuerza electromotriz de una pila formada por dicho semielemento y otro, $\mathrm{y}$ el potencial aislado de este otro semielemento. La fuerza electromotriz de una pila es fácil de medir, pero no asi el potencial aislado de un semielemento, lo cual resulta imposible hacer directamente. Lo que quiere decir que los potenciales aislados son relativos, no absolutos, y se expresan con referencia a un semielemento al que, por definición y convenio, y de una forma empírica y arbitraria, se le asigna un potencial igual a cero.

\section{medición potenciométrica de fuerzas electromotrices}

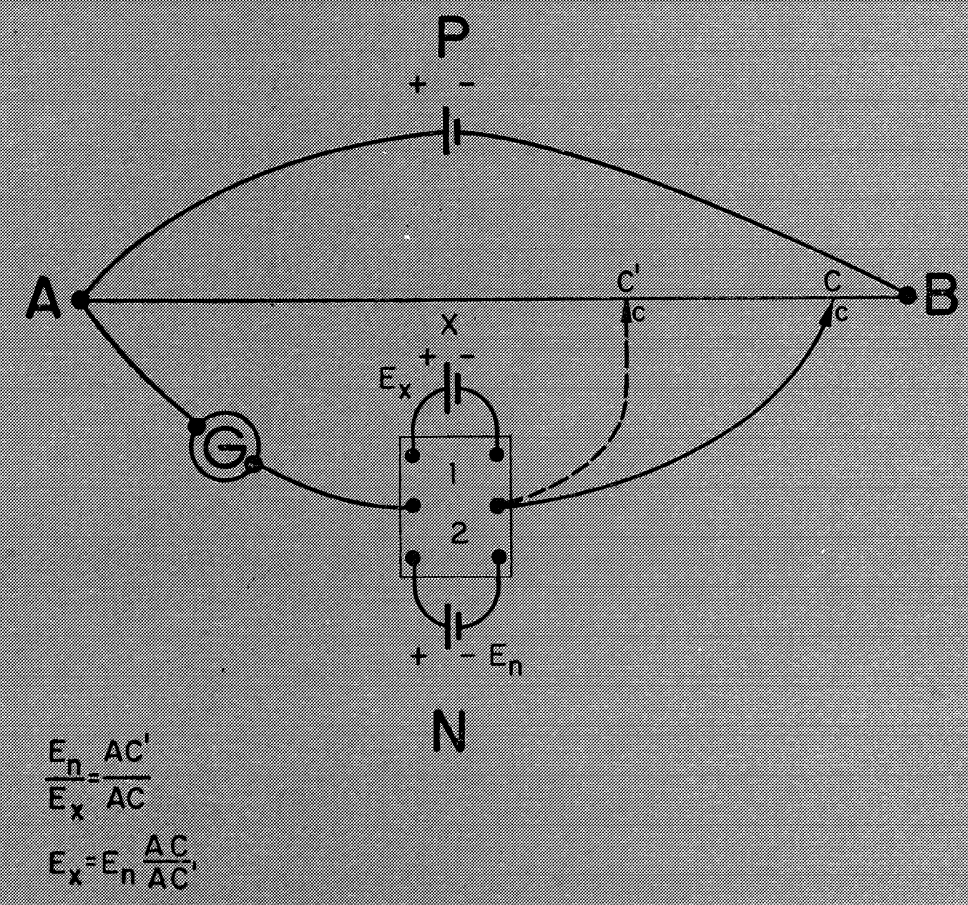

$P=P I L A$ DE TRABAJO.

$N=P I L A$ DE REFERENCIA.

$X=$ PILA DE F.E.M. DESCONOCIDA.

$G=$ GALVANOMETRO.

$A-B=$ PUENTE DE MEDIDA.

$c=$ CONTACTO, DESLIZANTE.

$C=$ POSICION DE COMPENSACION CORRESPONDIENTES AL CIRCUITO I.

$C^{\prime}=$ POSICION DE COMPENSACION CORRESPONDIENTES AL CIRCUITO 2.

$E_{n}=$ F.E.M. CONOCIDA Y CONSTANTE DE LA PILA DE REFERENCIA N.

$E=$ F.E.M. DE LA PILA X.

119.9.

\section{4. medida de fuerzas electromotrices y de potenciales aislados}

La fuerza electromotriz de una pila o elemento galvánico o voltaico se mide por comparación con la de otro, cuyo valor es conocido, empleándose el método de oposición o compensación (fig. 3).

El elemento o pila de referencia se llama elemento normal o patrón y tiene una fuerza 


\section{pila "Weston" normal}

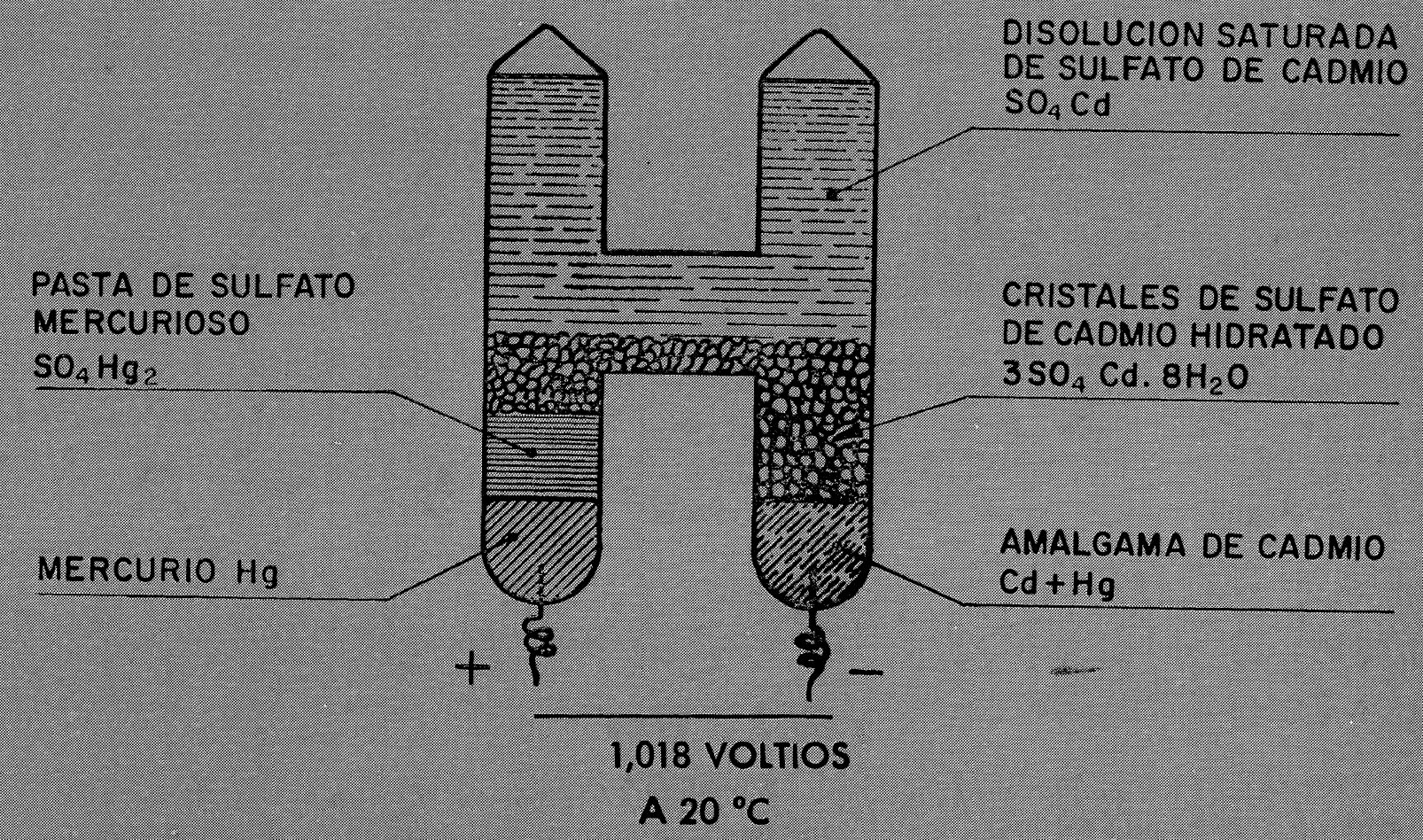

Fig. 4.

electromotriz constante, y prácticamente invariable (o muy poco variable) con la temperatura. Una de las más usuales es la pila Weston o de cadmio (1,018 voltios a $20^{\circ} \mathrm{C}$ ) (fig. 4$)$.

Los potenciales de los semielementos metálicos varían con la concentración iónica de la disolución o electrólito que contiene sus cationes, así como con la temperatura. Por ello se suelen dar los potenciales electrolíticos de la serie electromotriz referidos a unas condiciones normalizadas o "standard", tanto de temperatura (18 ó $25^{\circ} \mathrm{C}$, por ejemplo) como de concentración iónica (concentración unidad: disoluciones iónicas normales: un ion-gramo por litro de disolución). Los potenciales expresados en estas condiciones reciben el nombre de normales, y los semielementos a que corresponden se llaman también semielementos o electrodos normales. Estos potenciales, ordenados de menores (más negativos) a mayores (más positivos), reproducen la serie electromotriz de los metales (cuadro 2).

La medida práctica del potencial de un semielemento se lleva a cabo, como se ha indicado en 2.3, formando una pila con otro semielemento de referencia, cuyo potencial es cero por convenio, y midiendo la fuerza electromotriz de la pila así formada, por el método de compensación. En tales condiciones, la fuerza electromotriz medida es igual al potencial del semielemento en cuestión.

El semielemento o electrodo patrón de referencia al que se le asigna el potencial cero es el electrodo o semielemento normal de hidrógeno, constituido por una lámina de platino platinado sumergida en una disolución normal de un ácido, por la que burbujea hidrógeno gaseoso puro a presión de una atmósfera. 
Cuadro 2

POTENCIALES ELECTROLITICOS NORMALES A $25^{\circ} \mathrm{C}$ REFERIDOS

AL SEMIELEMENTO NORMAL DE HIDROGENO

\begin{tabular}{ll}
\multicolumn{2}{c}{ Elemento } \\
Litio & $\mathrm{Li}$ \\
Potasio & $\mathrm{K}$ \\
Calcio & $\mathrm{Ca}$ \\
Sodio & $\mathrm{Na}$ \\
Magnesio & $\mathrm{Mg}$ \\
Aluminio & $\mathrm{Al}$ \\
Manganeso & $\mathrm{Mn}$ \\
Zinc & $\mathrm{Zn}$ \\
Hierro & $\mathrm{Fe}$ \\
Níquel & $\mathrm{Ni}$ \\
Estaño & $\mathrm{Sn}$ \\
Plomo & $\mathrm{Pb}$ \\
Hidrógeno & $\mathrm{H}$ \\
Cobre & $\mathrm{Cu}$ \\
Plata & $\mathrm{Ag}$ \\
Mercurio & $\mathrm{Hg}$ \\
Oro & $\mathrm{Au}$
\end{tabular}

\begin{tabular}{|c|c|}
\hline Catión & Electrodo \\
\hline $\mathrm{Li}^{+}$ & $\mathrm{Li}+/ \mathbf{L i}$ \\
\hline $\mathrm{K}+$ & $\mathrm{K}+/ \mathrm{K}$ \\
\hline $\mathrm{Ca}^{2+}$ & $\mathrm{Ca}^{2} / \mathrm{Ca}$ \\
\hline $\mathrm{Na}+$ & $\mathrm{Na}+/ \mathrm{Na}$ \\
\hline $\mathrm{Mg}^{2+}$ & $\mathrm{Mg}^{2}+/ \mathbf{M g}$ \\
\hline $\mathrm{Al}^{3+}$ & $\mathrm{Al}^{3+/ \mathrm{Al}}$ \\
\hline $\mathbf{M n}^{2+}$ & $\mathrm{Mn}^{2+} / \mathrm{Mn}$ \\
\hline$Z \mathrm{n}^{2+}$ & $Z \mathrm{n}^{2}+/ Z \mathrm{n}$ \\
\hline $\mathrm{Fe}^{2+}$ & $\mathrm{Fe}^{2+} / \mathrm{Fe}$ \\
\hline $\mathrm{Ni}^{2+}$ & $\mathrm{Ni}^{2}+/ \mathrm{Ni}$ \\
\hline $\mathrm{Sn}^{2+}$ & $\mathrm{Sn}^{2+} / \mathrm{Sn}$ \\
\hline $\mathrm{Pb}^{2}+$ & $\mathbf{P b}^{2}+/ \mathbf{P b}$ \\
\hline $\mathbf{H}+$ & $\mathrm{H}+/ \mathrm{H}_{2}(\mathrm{Pt}$ \\
\hline $\mathrm{Cu}^{2+}$ & $\mathrm{Cu}^{2}+/ \mathrm{Cu}$ \\
\hline $\mathrm{Ag}^{+}$ & $\mathrm{Ag}+/ \mathrm{Ag}$ \\
\hline $\mathrm{Hg}_{2}^{2}+$ & $\mathrm{Hg}_{2}^{2}+/ \mathrm{Hg}$ \\
\hline $\mathrm{Au}^{3+}$ & $\mathrm{Au}^{3}+/ \mathrm{Au}$ \\
\hline
\end{tabular}

$\rightarrow \mathrm{Me}$

\begin{tabular}{|c|c|c|}
\hline Proceso & \multicolumn{2}{|c|}{ Potencial } \\
\hline $\mathrm{Li}^{+}+\mathrm{e}^{-} \rightarrow \mathrm{Li}$ & $-2,96$ & voltios \\
\hline $\mathrm{K}^{+}+\mathrm{e}^{-} \rightarrow \mathrm{K}$ & $-2,92$ & ” \\
\hline $\mathrm{Ca}^{2}++2 \mathrm{e}^{-} \rightarrow \mathrm{Ca}$ & $-2,86$ & ” \\
\hline $\mathrm{Na}^{+}+\mathrm{e}^{-} \rightarrow \mathrm{Na}$ & $-2,71$ & " \\
\hline $\mathrm{Mg}^{2}++2 \mathrm{e}^{-} \rightarrow \mathrm{Mg}$ & $-1,87$ & " \\
\hline $\mathrm{Al}^{3}++3 \mathrm{e}^{-} \rightarrow \mathrm{Al}$ & $-1,35$ & " \\
\hline $\mathrm{Mn}^{2}++2 \mathrm{e}^{-} \rightarrow \mathrm{Mn}$ & $-1,18$ & ” \\
\hline$Z^{2}++2 e^{-} \rightarrow Z n$ & $-0,76$ & ” \\
\hline $\mathrm{Fe}^{2+}+2 \mathrm{e}^{-} \rightarrow \mathrm{Fe}$ & $-0,44$ & , \\
\hline $\mathrm{Ni}^{2}++2 \mathrm{e}^{-} \rightarrow \mathrm{Nj}$ & $-0,22$ & ” \\
\hline $\mathrm{Sn}^{2}++2 \mathrm{e}^{-} \rightarrow \mathrm{Sn}$ & $-0,14$ & ” \\
\hline $\mathrm{Pb}^{2}++2 \mathrm{e}^{-} \rightarrow \mathrm{Pb}$ & $-0,13$ & " \\
\hline $2 \mathrm{H}++2 \mathrm{e}^{-} \rightarrow \mathrm{H}_{2}$ & $\mp 0,00$ & ” \\
\hline $\mathrm{Cu}^{2}++2 \mathrm{e}-\rightarrow \mathrm{Cu}$ & $+0,34$ & , \\
\hline $\mathrm{Ag}^{+}+\mathrm{e}^{-} \rightarrow \mathbf{A g}$ & $+0,80$ & $"$ \\
\hline $\mathrm{Hg}_{2}^{2}++2 \mathrm{e}^{-} \rightarrow 2 \mathrm{Hg}$ & $+0,86$ & $"$ \\
\hline $\mathrm{Au}^{3+}+3 \mathrm{e}^{-} \rightarrow \mathrm{Au}$ & $+1,50$ &.$”$ \\
\hline
\end{tabular}

Por lo tanto, el potencial normal de un semielemento cualquiera es igual a la fuerza electromotriz de la pila formada por el semielemento normal en cuestión y el semielemento normal de hidrógeno.

Aparte de éste existen otros semielementos o electrodos de referencia, patrones secundarios, de mucho más fácil realización y manejo, entre los que destacan los electrodos de calomelanos, normal, decimonormal y saturado, y sobre todo este último (fig. 5).

El semielemento de calomelanos saturado está constituido tal como indica la figura 5, en una de sus múltiples versiones. Recibe el nombre de saturado porque el líquido electrolítico es una disolución saturada de cloruro potásico.

La medida del potencial aislado de un semielemento $E$ (metal introducido en un electrólito) se hace potenciométricamente, de acuerdo con el esquema de la figura 6. Para ello se forma un elemento o pila $X$ con el semielemento de referencia (calomelanos saturado) y el semielemento de potencial desconocido. En la posición de circuito 1, de oposición entre la pila $P$ y la pila Weston $N$ se compensa con el reostato variable $V_{1}$ hasta que no pase corriente por el galvanómetro $C$, con lo que, dado el valor de la resistencia fija $F$ que consume exactamente 0,5 voltios de los 1,5 voltios de la pila seca $P$, entre los extremos fijos $a$ y $b$ del potenciómetro se tiene una diferencia de potencial igual a la fuerza electromotriz de la pila Weston $(1,018)$, esto es, prácticamente 1 voltio. El reostato $V_{2}$, que puede ser un hilo calibrado, está dividido y marcado de forma que se conoce la diferencia de potencial entre su extremo fijo $a$ y su extremo variable $c$, pues si entre $a$ y $b$ hay un metro de hilo dividido en milímetros, al que corresponde un voltio una vez hecho el ajuste anterior, a cada milímetro corresponderá un milivoltio. En la posición de circuito 2 de oposición entre la pila $P$ y la pila $X$, se compensa con el reostato variable o hilo calibrado $V_{2}$ hasta que no pase corriente por el galvanómetro $G$, con lo que, entre el extremo fijo $\sigma$ y la posición del 


\section{semielemento o electrodo de "calomelanos saturado"}

patrón secundario para la medida de potenciales aislados

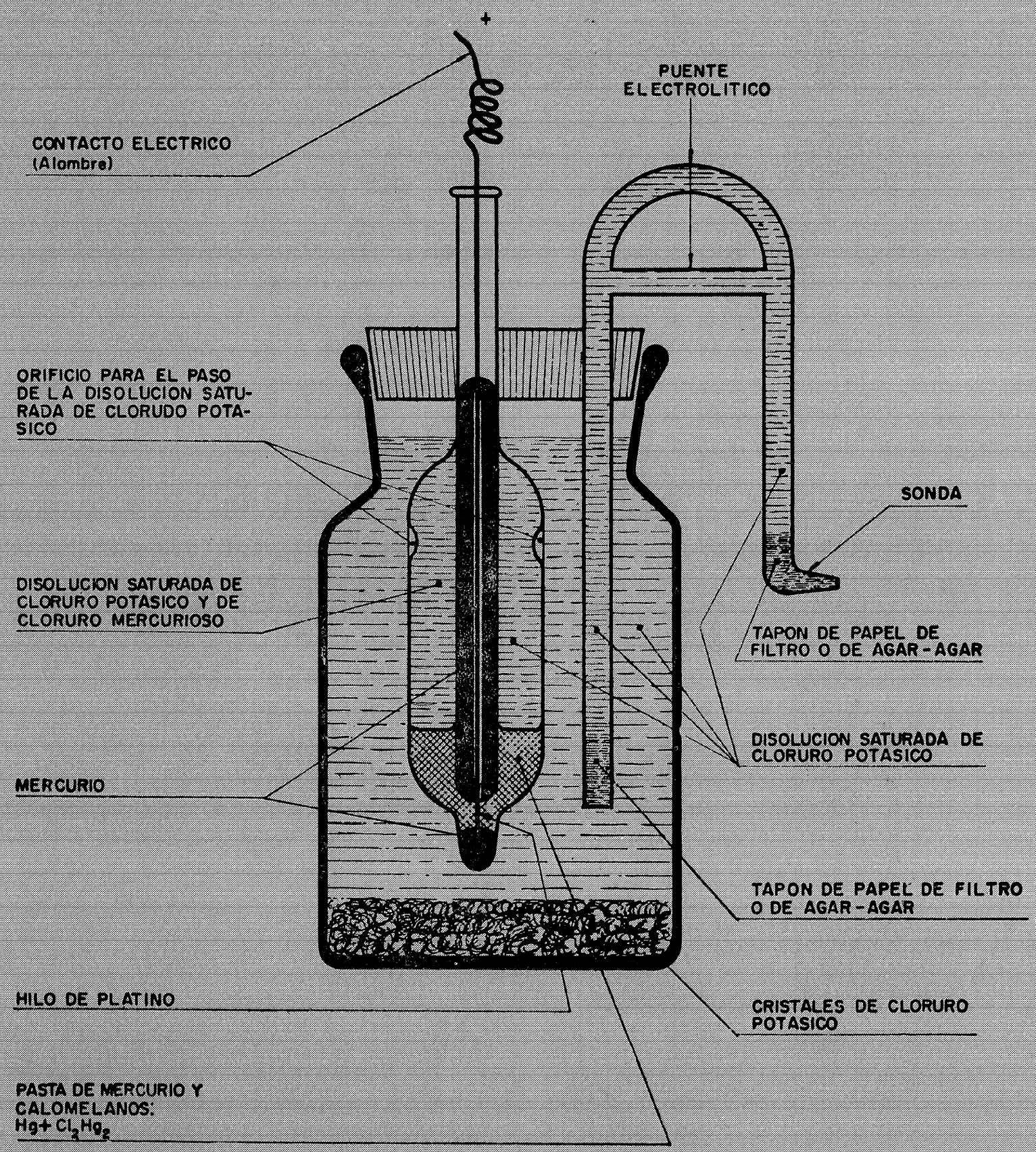

Fig. 5 .

cursor $c$ del potenciómetro se tiene una diferencia de potencial igual a la fuerza electromotriz de la pila $X$. Si a la longitud $a b$ de hilo calibrado correspondia una diferencia de potencial de 1 voltio, a la longitud $a c$ que es la que ahora actúa le corresponderá $a c / a b$ voltios. En los distintos puntos $c$ variables del hilo suelen estar señalados los correspon- 


\section{medición potenciométrica de potenciales aislados}

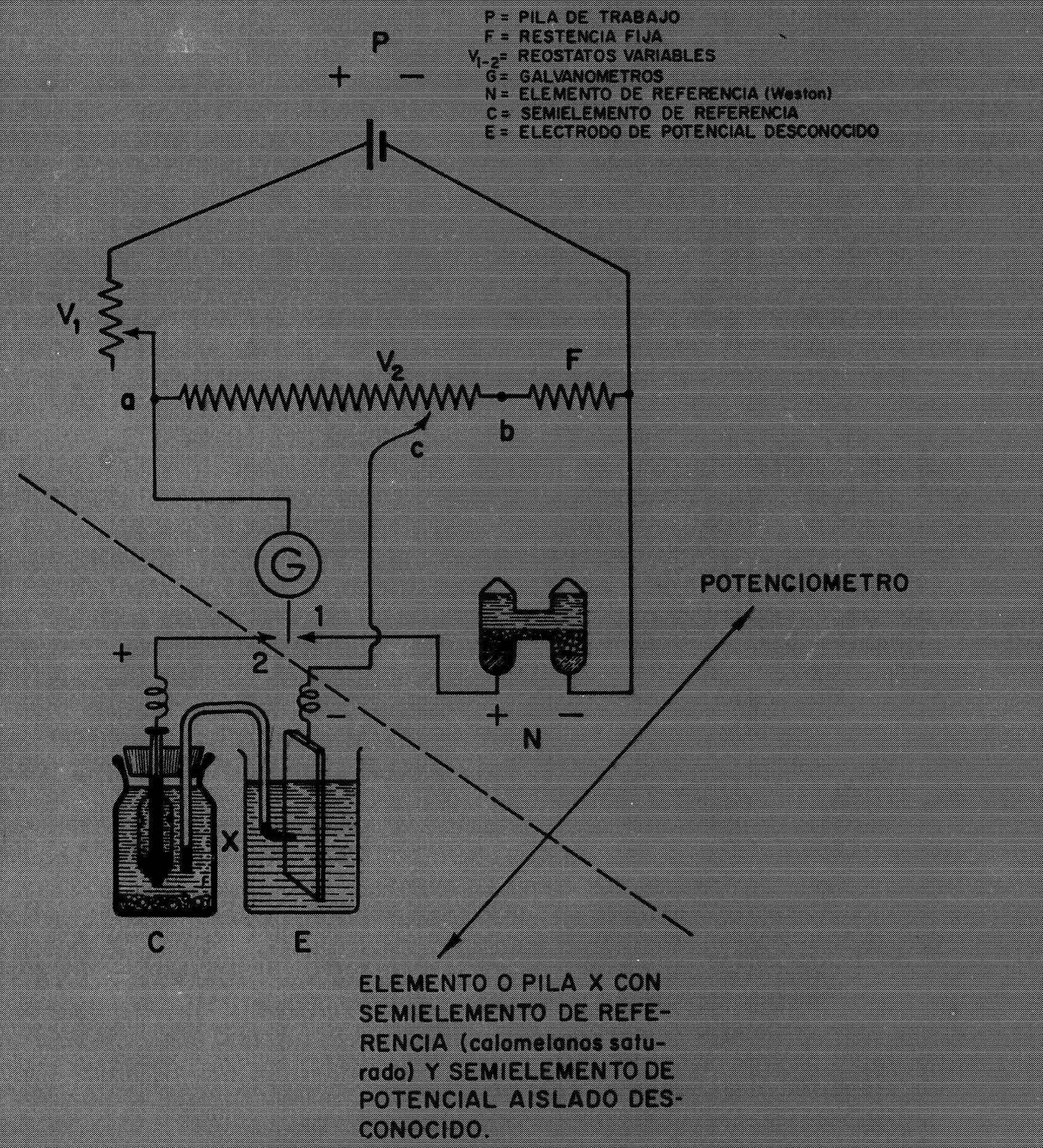

Fis. 0.

dientes valores $a c / a b$, es decir, se puede leer directamente la diferencia de potencial o fuerza electromotriz de los elementos problema $X$.

Este voltaje $V$ leído es la diferencia algebraica entre el potencial del electrodo $E$ y el 
potencial del electrodo de referencia $C: V=E-C$, con lo que, asignando arbitrariamente el valor cero al potencial del semielemento de referencia, el voltaje leído $V$ da directamente el potencial del semielemento $E: V=E$.

Conocido (o determinado de una vez para siempre) el potencial de los electrodos de referencia respecto del electrodo normal de hidrógeno, es fácil calcular, con relación a éste tomado como cero, el potencial de cualquier electrodo, una vez medido su potencial frente al electrodo de referencia. Basta para ello sumar algebraicamente al potencial, $V$, medido, el potencial del electrodo de referencia respecto del normal de hidrógeno. Por ejemplo, el potencial del electrodo de calomelanos saturado frente al normal de hidrógeno es $+0,2415$ voltios; pues bien, si es $E_{c}$ el potencial de un semielemento cualquiera respecto del electrodo de calomelanos saturado, el potencial de dicho semielemento frente al electrodo normal de hidrógeno $E_{h}$ será :

$$
E_{h}=E_{c}+0,2415 \text { voltios, }
$$

considerando $E_{c}$ con su signo. Es decir, el potencial de un semielemento respecto del electrodo normal de hidrógeno tomado como cero es la suma algebraica de su potencial respecto de un semielemento cualquiera, y el potencial de éste respecto del semielemento normal de hidrógeno.

En cuanto al signo de los potenciales, es una cuestión puramente convencional. En el cuadro 2 se han considerado los procesos de reducción de los metales (ganancia de electrones): $\mathrm{Me}^{n+}+n e^{-} \rightarrow M e$, por lo que los correspondientes potenciales de equilibrio (o reversibles) de un metal $M e$ en contacto con sus iones $M e^{n+}$ son los potenciales de reducción. Los potenciales de los procesos de oxidación inversos (pérdida de electrones) de los metales

$$
M e \rightarrow M e^{n+}+n e^{-}
$$

son los correspondientes potenciales de oxidación que, en el equilibrio (en condiciones de reversibilidad) son iguales y de signo contrario a los anteriores. Por lo demás, como se ha indicado en 2.3, se suele adoptar como signo del potencial el que toma el metal de acuerdo con el proceso al que está sometido: si es de reducción (ganancia de electrones), negativo; si es de oxidación (pérdida de electrones), positivo. En todo caso se suele adoptar con carácter general (aunque con excepciones) el criterio de que el potencial normal del zinc (extremo superior de la serie electromotriz) es negativo.

\section{5. corrosión electroquimica: elementos o pilas de corrosión}

Un ejemplo de corrosión electroquímica lo constituye el ataque y disolución de los metales por líquidos electrolíticos (en particular, por ácidos y bases) :

$$
\begin{gathered}
\mathrm{Fe}+2 \mathrm{H}^{+} \rightarrow \mathrm{Fe}^{2+}+\mathrm{H}_{2} \\
\mathrm{Zn}+2 \mathrm{H}^{+} \rightarrow \mathrm{Zn}^{2+}+\mathrm{H}_{2} \\
\mathrm{Zn}+2 \mathrm{OH}^{-} \rightarrow \mathrm{ZnO}_{2}^{2-}+\mathrm{H}_{2} \\
\mathrm{Al}+2 \mathrm{OH}^{-}+2 \mathrm{H}_{2} \mathrm{O} \rightarrow 2 \mathrm{AlO}_{2}^{-}+3 \mathrm{H}_{2}
\end{gathered}
$$

Estas reacciones, según otro punto de vista, se consideran como representativas de corrosiones químicas (hierro o zinc atacados por ácido sulfúrico o clorhídrico, y aluminio o zinc atacados por hidróxido sódico concentrado). No lo son, sin embargo, más que en apariencia, porque para que de hecho lo fuesen, habría de darse el caso ideal de que todos los átomos de la superficie del metal fuesen igualmente susceptibles al ataque por el electrólito, y éste tuviese las mismas posibilidades de acceso a ellos, cosa que casi nunca sucede en la práctica. 
En realidad, la reacción

$$
\mathrm{SO}_{4} \mathrm{H}_{2}+\mathrm{Zn} \rightarrow \mathrm{SO}_{4} \mathrm{Zn}+\mathrm{H}_{2}
$$

no transcurre, o lo hace con gran lentitud y dificultad, cuando se trata de zinc muy puro, lo que indica que las impurezas de otros metales son las responsables de la rápida disolución del zinc.

Otro tanto sucede cuando el zinc se pone en contacto eléctrico con un metal noble (platino, por ejemplo) dentro del electrólito: el hidrógeno se desprende sobre el metal noble del mismo modo que antes se desprendía sobre los metales que impurifican ordinariamente al zinc.

Supuesto que uno de estos metales sea el cobre, tiene lugar un proceso análogo al de funcionamiento de la pila de Volta (o la de Daniell), y, en general, de los elementos voltaicos o galvánicos primarios (fig. 1a). El proceso consiste en la oxidación o disolución iónica (corrosión) del zinc que, como metal menos noble o de potencial más negativo (de mayor presión de disolución electrolítica), funciona como ánodo, acompañada de desprendimiento de hidrógeno (reducción de hidrogeniones $\mathrm{H}^{+} \mathrm{o}$ de iones hidronio $\mathrm{H}_{3} \mathrm{O}^{+}$) sobre el cobre, el cual, como metal más noble o de potencial más positivo (de menor presión de disolución electrolítica), se comporta como cátodo. Las reacciones en ambos electrodos son:

$$
\begin{aligned}
& \text { Anodo }(-): \mathrm{Zn} \rightarrow \mathrm{Zn}^{2+}+2 e^{-} \\
& \text {Cátodo (+) }: 2 \mathrm{H}^{+}+2 e^{-} \rightarrow \mathrm{H}_{2} \uparrow \text { (Volta) } \\
& \mathrm{Cu}^{2+}+2 e^{-} \rightarrow \mathrm{Cu} \text { (Daniell) }
\end{aligned}
$$

y el esquema eléctrico a que se ajustan responde a las figuras 7 y 8 , cumpliéndose en todo caso las leyes de Ohm y de Faraday.

Las correspondientes reacciones en el caso del hierro son:

$$
\begin{aligned}
& \text { Anodo }(-): \mathrm{Fe} \rightarrow \mathrm{Fe}^{2+}+2 e^{-} \\
& \text {Cátodo }(+): 2 \mathrm{H}^{+}+2 e^{-} \rightarrow \mathrm{H}_{2} \uparrow
\end{aligned}
$$

Según el equilibrio de disociación iónica del agua

$$
4 \mathrm{H}_{2} \mathrm{O} \rightarrow 2 \mathrm{H}_{3} \mathrm{O}^{+}+2 \mathrm{OH}^{-}
$$

la supresión de iones hidronio $\mathrm{H}_{3} \mathrm{O}^{+}$(protones e hidrogeniones $\mathrm{H}^{+}$hidratados) por descarga y neutralización:

$$
2 \mathrm{H}_{3} \mathrm{O}^{+}+2 e^{-} \rightarrow 2 \mathrm{H}_{2} \mathrm{O}+2 \mathrm{H}
$$

y el desprendimiento de hidrógeno gaseoso (molecular):

$$
2 \mathrm{H} \rightarrow \mathrm{H}_{2} \uparrow
$$

deja un exceso de dos iones oxhidrilo $2 \mathrm{OH}^{-}$libres, pues la suma de los tres procesos $[\mathrm{X}]$, [XI] y [XII] equivale a la reacción global:

$$
4 \mathrm{H}_{2} \mathrm{O}+2 e^{-} \rightarrow 2 \mathrm{H}_{2} \mathrm{O}+2 \mathrm{OH}^{-}+\mathrm{H}_{2} \uparrow
$$

es decir:

$$
2 \mathrm{H}_{2} \mathrm{O}+2 e^{-} \rightarrow 2 \mathrm{OH}^{-}+\mathrm{H}_{2} \uparrow
$$

Esto permite explicar también el ataque del hierro por las sales neutras como, por ejemplo, los cloruros alcalinos o el cloruro cálcico: 


$$
\text { Anodo }(\rightarrow): \mathrm{Fe} \rightarrow \mathrm{Fe}^{2+}+2 e^{-} ; \text {Cátodo }(+): \begin{aligned}
& 2 \mathrm{Na}^{+}+2 e^{-} \rightarrow 2 \mathrm{Na} \\
& 2 \mathrm{Na}+2 \mathrm{H}_{2} \mathrm{O} \rightarrow 2 \mathrm{NaOH}+\mathrm{H}_{2} \uparrow 2 \mathrm{NaOH} \rightarrow 2 \mathrm{Na}^{+}+2 \mathrm{OH}^{-} \\
& \frac{2 \mathrm{H}_{2} \mathrm{O}+2 e^{-} \rightarrow 2 \mathrm{OH}^{-}+\mathrm{H}_{2} \uparrow}{}
\end{aligned}
$$

La sola diferencia está en que, siendo única la reacción anódica primaria (disolución del hierro en forma iónica divalente), el complejo de reacciones catódicas puede ser distinto, para llegar al mismo resultado final: desprendimiento de hidrógeno con consumo local de hidrogeniones $\mathrm{H}^{+}$o iones hidronio $\mathrm{H}_{3} \mathrm{O}^{+}$y consiguiente exceso, también local, de iones oxhidrilo $\mathrm{OH}^{-}$(alcalinidad).

A veces puede explicarse el resultado de los procesos finales idénticos [XIV] y [XVIII], por disolución catódica de oxígeno, reacción que contrarrestaría a la de desprendimiento de hidrógeno (despolarización):

$$
\begin{gathered}
1 / 2 \mathrm{O}_{2} \rightarrow \mathrm{O} \\
\mathrm{O}+\mathrm{H}_{2} \mathrm{O}+2 e^{-} \rightarrow 2 \mathrm{OH}^{-}
\end{gathered}
$$

Pero, para que haya reacción en los electrodos y, por lo tanto, proceso anódico de corrosión, en el caso de un elemento galvánico primario, es preciso cerrar el circuito poniendo en contacto eléctrico externo el ánodo con el cátodo (figs. 7 y 8) a fin de que los electrones que se liberan en el primero puedan tomar parte en la reacción catódica del segundo. La corrosión anódica queda así supeditada a tal contacto y, por consiguiente, a la circulación de electrones desde el ánodo hasta el cátodo (o de corriente eléctrica entre el cátodo y el ánodo), siendo más extensa la corrosión cuanto mayor es la cantidad de electricidad (culombios: amperios por segundo) que circula, y tanto más intensa cuanto mayor es la densidad de corriente anódica (amperios o miliamperios por metro, decímetro o centimetro cuadrado de superficie anódica).

En el caso de un metal sometido a corrosión existe ya contacto eléctrico interno entre zonas anódicas y catódicas, y la corrosión se produce al simple contacto con el electrólito. Estas zonas se crean, bien sea por la presencia de impurezas de metales de distinta nobleza que el metal base, o bien por heterogeneidades superficiales del mismo debidas a diversas causas (incluso mecánicas), que hagan comportarse a unos puntos como cátodos con respecto a otros.

Pero ni siquiera es necesaria la existencia de una heterogeneidad interna; basta con que ésta sea externa para que, entre dos metales absolutamente iguales, o entre dos zonas idénticas de un mismo metal, unas se constituyan en ánodos y otras en cátodos. Esta heterogeneidad externa viene dada en algunos casos por diferencias de concentración del electrólito (pilas de concentración) y en otros por diferencias de facilidad y de velocidad de acceso de oxígeno o de aire a distintas zonas de un metal. En tales circunstancias las zonas de más fácil acceso se convierten en cátodos, quedando así relativamente más protegidas contra la corrosión, ya que en ellas el oxígeno modifica el potencial en el sentido que corresponde, según uno de los procesos catódicos antes considerados $[\mathrm{XX}]$, o bien:

$$
2 \mathrm{H}_{3} \mathrm{O}^{+}+2 e^{-} \rightarrow 2 \mathrm{H}_{2} \mathrm{O}+2 \mathrm{H} ; \quad 2 \mathrm{H}+\mathrm{O} \rightarrow \mathrm{H}_{2} \mathrm{O}
$$
constituyendo este último proceso una despolarización y favoreciendo la reacción catódica.

Las zonas más inaccesibles para el aire o el oxigeno se convierten, por el contrario, en ánodos en los que la corrosión se desarrolla. Cuanto mayor y más rápido y continuo sea el aporte de oxígeno a las zonas catódicas, mayor protección recibirán éstas, pero mayor 


\section{pilg de Volta}

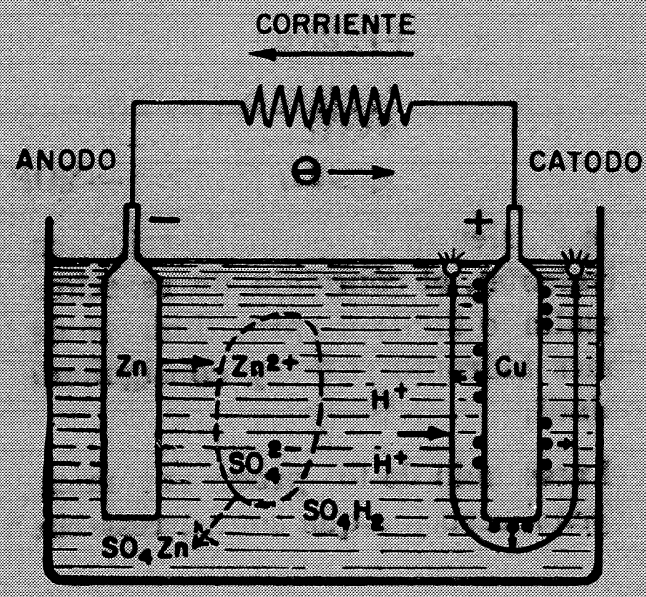

$$
\begin{aligned}
& \text { ANODO }(-): \mathrm{Zn} \rightarrow \mathrm{Zn}^{2+}+2 \overline{\mathrm{e}} \quad \text { CATODO }(+): 2 \mathrm{H}^{+}+2 \mathrm{e} \rightarrow \mathrm{H}_{2} \\
& \text { PROCESO TOTAL: } \mathrm{Zn}+2 \mathrm{H}^{+}=\mathrm{Zn}^{2}++\mathrm{H}_{2} \mathrm{I}
\end{aligned}
$$

\section{pila de Daniell}

Fig. 7.

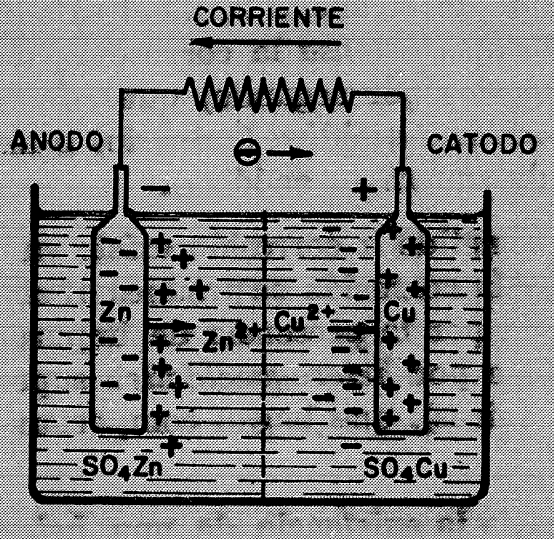

$$
\begin{aligned}
& \text { ANODO (-): } Z n \rightarrow Z^{2+}+2 \overline{C B} \quad \text { CATODO }(+): \mathrm{Cu}^{2+}+2 \bar{e} \rightarrow C u \\
& \text { PROCESO TOTAL: } Z n+C u^{2+}=Z n^{2+}+C u
\end{aligned}
$$

Iig. 8.

será también el avance del proceso anódico y, por lo tanto, la corrosión, puesto que más intensa será la circulación de corriente (o de electrones) por la pila así formada, la cual recibe el nombre de elemento de aireación diferencial.

La única diferencia entre las pilas galvánicas y las de corrosión es de escala u orden de magnitud: los elementos voltaicos son macrocélulas y las pilas locales o elementos de corrosión son microcélulas. 
En definitiva, la circulación de electrones o de corriente eléctrica entre dos metales o aleaciones, o entre dos puntos próximos de un mismo metal o aleación en contacto con un electrólito, requiere, por una parte, la existencia de una diferéncia de potencial entre ellos, es decir, que cada uno se encuentre a un potencial distinto. Por otra parte, para la más fácil circulación de esa corriente es preciso que el electrólito tenga una buena conductividad eléctrica.

Las diferencias de potencial de los distintos metales frente a sus disoluciones iónicas normales constituyen los potenciales normales de los respectivos metales (cuadro 2) que, de menor a mayor, con su signo, es decir, desde los potenciales negativos de gran valor absoluto hasta los positivos de gran valor absoluto, pasando pör los potenciales próximos a cero, tanto negativos como positivos, e incluso por el potencial cero (convencional), esto es, el corresponddientè a un electrodo metálico inerte (platino platinado) sumergido en una disolución normal de hidrogeniones, dan lugar a la serie electroquímica o serie electromotriz de los metales. Los elementos situados a la cabeza de la serie son los menos nobles o más fácilmente corrosibles (de potencial más electronegativo), en tanto que los situados $\therefore$ a la cola son los más nobles e inerte, o'menos corrosibles (de potencial más electropositivo). Los primerós tenderán a desplazar a los segundos de sus disoluciones $\mathbf{y}$, por lo tanto, los situados por encima del hidrógenó (de potencial normal cero) serán atacados por los ácidos con dẹsprendimiento de hídrógeno.

Lo que antecede explica por quéla corrosión general o uniforme rara vez se da de una forma completa, pues queda restringida a los casos de corrosiön puramente química, mientras que, por el contrario, en la práctica son muy frecuentes los casos de corrosión localizada, adscritos siempere a procesos de corrosión electroquimica en su sentido más amplio, siendo estos casos, por desgracia, los más temibles por sus consecuencias.

Finalmente, todo esto permite también comprender el mecanismo de la protección catódica, consistente en convertir en cátodo todà la superficie del metal que se quiere proteger; a costa del sacrificio de ánodos constituidos por metales menos nobles, con potenciales mucho más electronegativos que el correspondiente al metal protegido; en las condiciones de servicio de éste, o bien por la aplieación al metal de una corriente externa de tal naturaleza que el metal adquiera un potencial tal que lo convierta manifiestamente en cátodo en las condiciones de servicio $(2.6)$,

\subsection{1. tipos de corrosión electroquímica}

La corrosión electroquímica o localizada, asentada siempre sobre heterogeneidades o disimetrias, puede' ser" de "varios tipos y obedecer a diversas causas. Así se da:

I) la corrosión intercristalina o fisuranté, sin manifestación externa visible, la cual puede dar lugar a roturas frágiles, y que se presenta sin necesidad de que existan tensiones externas de tracción; sin embargo, estas ténsiones pueden contribuir a acelerar la corrosión intercristálina, ya que tienden a agrandar las fisuras y a exponer a la corrosión electrolítica nuevas superficies metálicas particularmente sensibles a ella;

II) la corrosión selectiva, que se da en aleaciones (disoluciones sólidas) con tendencia al ataque de uno solo de los constituyentes de las mismas (zinc de los latones) y a la producción de fisuras;

III) la corròsión bajo tensión, que es una combinación de corrosión y de tensiones estáticas residuales o externas, sin las cuales, a diferencia de la corrosión inter- 
cristalina, no se presenta, pero que, sin embargo, puede adoptar el carácter de corrosión intercristalina, siendo observable en las aleaciones;

IV) la corrosión alveolar ("pitting”), que es independiente de la estructura de los cristales del metal y se da con gran frecuencia, siendo un ejemplo de ello el ataque del hierro en atmósfera húmeda, y que, si bien es una corrosión localizada, los puntos de corrosión se distribuyen al azar sobre la superficie del metal;

V) la corrosión por fatiga;

VI) la corrosión por contactos galvánicos (formación de macrocélulas);

VII) la corrosión por corrientes vagabundas. En este último caso las zonas anódicas y catódicas del metal que se corroe están inducidas por una corriente o fuerza electromotriz externa, y en lugar de formarse una pila, elemento o célula galvánica se forma una célula electrolítica (fig. 1b). En ella el ánodo constituye el polo positivo, por donde entra la corriente (es decir, por donde ésta abandona el metal para entrar en el electrólito), y el cátodo, el polo negativo, por donde la corriente sale del electrólito para pasar al metal; la circulación de electrones tiene lugar en sentido contrario.

\section{6. potencial y corrosión}

Los potenciales de la serie electromotriz normal no son sino un reflejo de la energía potencial de los elementos en estado metálico (reducido) o con valencia nula, frente a la energía potencial de los mismos en estado iónico (oxidado) o con valencias positivas (ca. tiones).

Cuanto más electronegativo es el potencial de un metal en contacto con una disolución de sus iones, tanto mayor es la tendencia a la corrosión de dicho metal (extremo superior de la serie electromotriz de los metales); por el contrario, cuanto más electropositivo es dicho potencial, tanto menor es la tendencia a la corrosión del metal mencionado (extremo inferior de la citada serie).

Así, pues, el valor del potencial de un metal en unas condiciones dadas determina el que el metal se corroa o no en esas condiciones. Los metales suelen presentar dos series de valores distintos del potencial, más o menos bien delimitadas, y que con arreglo a lo indicado implican dos comportamientos distintos y aun opuestos del metal: un comportamiento activo, con disolución (ataque y corrosión) del metal, y otro pasivo, con producción de otros fenómenos, pero sin disolución ni corrosión metálica. Naturalmente que los potenciales activos o correspondientes al estado activo de los metales son, en cada caso, los más electronegativos, en tanto que los potenciales pasivos o correspondientes al estado pasivo son los más electropositivos.

La pasividad de los metales es, pues, un estado de garantía o protección contra la corrosión de los mismos, y al que se debe tender artificialmente cuando dicho estado no se da de una forma espontánea y natural en unas condiciones determinadas.

Cabe también considerar los potenciales de óxido-reducción, que son los correspondientes al proceso de paso de un anión o catión de un grado de valencia a otro distinto, con pérdida de electrones (oxidación) o ganancia de electrones (reducción), en cada caso. Estos potenciales dependen, además, de las concentraciones iónicas en las formas oxidada y reducida, y se definen y valoran para unas concentraciones dadas y en condiciones de reversibilidad de proceso. 


\section{3. mecanismo de la corrosión de armaduras en el hormigón}

La corrosión metálica en general, y la del hierro en el hormigón en particular, es considerada a veces como un "fenómeno aleatorio" en el que el potencial del metal varía de una forma errática, fijándose o no, y, en el caso de fijarse, haciéndolo durante más o menos tiempo, bien sea en la región de pasividad, o bien en la de la corrosión activa, o bien en la de óxido-reducción reversible.

Ello es debido a que el hierro está casi en equilibrio con el agua en circunstancias normales y ordinarias, es decir, a que la energía libre de la reacción entre ambos es muy pequeña. Por ello, el hierro frente al agua es siempre más o menos pasivo en las citadas circunstancias, y casi nunca total y perfectamente activo, y por eso su potencial es caprichosamente variable e inestable.

Los "seudo-potenciales" inestables que en tales condiciones se miden tienen un carácter tanto más aleatorio cuanto más profunda es la pasividad, y recíprocamente: la observación de potenciales muy variables lleva a pensar, en primer lugar, en un fenómeno de pasividad.

Si se hace extensiva esta idea al caso de metales distintos, se llega a la conclusión de que la serie electromotriz normal de los metales no debe utilizarse para predecir el comportamiento de éstos desde el punto de vista de la corrosión, sino que, en su lugar, deben utilizarse las series galvánicas prácticas, y, aun así, no siempre hay garantía de coincidencia entre la predicción y la realidad, como demuestra sobradamente la experiencia. En todo caso la probabilidad de corrosión por formación de pilas galvánicas será tanto menor cuanto más próximos estén los metales en las series y cuanto mayor sea la relación superficial de ánodo a cátodo, para que la densidad de corriente anódica sea lo menor posible.

En el caso del hierro, los potenciales próximos a cero * son los más estables y representativos, pero resultan peligrosos desde el punto de vista de la corrosión, tanto si se consiguen natural como artificialmente, pues de existir una zona mucho más negativa en el mismo metal, se establecería una corriente de corrosión. Por otra parte, potenciales estables no se observan más que en medios alcalinos puros exentos de iones salinos (cal pura) y son los que se dan en la protección catódica del hierro, por apartamiento o ausencia de aniones salinos (concretamente del anión cloruro $\mathrm{Cl}^{-}$).

En el hormigón de cemento portland, que es un medio de pH 12 teóricamente, no se observan estos potenciales tan estables, sino otros correspondientes a la zona de pasividad. Así, pues, la alcalinidad de los hormigones supone una circunstancia ventajosa para la protección de las armaduras, pues a causa de ella el hormigón se comporta frente al acero como un inhibidor anódico, ya que da lugar a la deposición de un recubrimiento protector sobre el metal y dificulta la difusión de oxígeno hacia el mismo, impidiendo la reacción catódica normal y, por lo tanto, el proceso completo, incluida la reacción anódica de corrosión.

Pero, si por una circunstancia cualquiera, el $\mathrm{pH}$ disminuye, puede alcanzarse un potencial activo de disolución o corrosión del hierro. Los aniones salinos, y concretamente el anión cloruro $\mathrm{Cl}^{-}$, pueden, por regreso a las zonas catódicas después de una interrupción de la corriente de protección catódica cuando se utiliza este método protector, o por disminución del $\mathrm{pH}$, dar al traste con la pasividad y provocar la corrosión de las armaduras.

* Potenciales referidos al semielemento de calomelanos saturado.

(Continuará.) 